\title{
A. Recherches anatomopathologiques chez le rat ingérant différentes doses d'huile d'arachide ou d'huile de colza à faible teneur en acide érucique (Huile de colza Primor).
}

\section{5a. Etude histologique du myocarde}

\author{
par R. CLUZAN, M. SUSCHETET *, G. ROCQUELIN *, R. LEVILLAIN \\ SERMEP, 38, rue Léo-Lagrange, 94270 Kremlin Bicêfre. \\ * Station de Recherches sur la Qualité des Aliments de l'Homme, I.N.R.A. \\ 7, rue Sully, 21034 Dijon Cédex.
}

Summary. A. Anatomo-pathological research on rats ingesting different doses of peanut oil or of low-erucic acid rapeseed oil (Primor rapeseed oil). 5a. Histology of the myocardium.

The number of rats with lesions did not significantly vary from one group to the ofher. With the 15 p. 100 oil diet, the Primor rapeseed oil had a more cardiopathogenic effect than peanut oil. After 1 year of the 5 p. 100 diet, the Primor rapeseed oil lot tended to present more lesions than the peanut oil lot.

Les détails concernant les techniques employées pour la préparation des coupes du myocarde ont été donnés précédemment.

\section{Matériel et méthodes.}

Pour chaque coupe, on a apprécié : le nombre de lésions, le volume de la lésion nécrotique $(V)$, l'intensité de la réaction histiocyłaire $(G)$, le siège de la lésion selon la place dans le myocarde (sub-épicarde, mésocarde, sous-endocarde).

Le volume $(V)$ de la lésion nécrotique a été coté, selon son importance, de la façon suivante : $V_{1}=$ lésion correspondant à un diamètre de 1 à 5 cellules myocardiques ; $V_{2}=$ lésion correspondant à un diamètre de 6 à 20 cellules myocardiques ; $V_{3}=$ lésion correspondant à un diamètre de plus de 20 cellules myocardiques.

L'intensité de la réaction granulomateuse $(G)$ a été cotée, selon son importance, de la façon suivante : $G_{1}=3$ à 4 éléments macrophagiques; $G_{2}=5$ à 20 éléments macrophagiques ; $G_{3}=$ plus de 20 éléments macrophagiques. Certaines lésions, d'interprétation difficile, ont été classées « douteuses".

Analyse statistique des résultats.

Les fréquences d'animaux porteurs de lésions cardiaques, en fonction de la nature et/ou de la dose d'huile dans le régime, ont été comparées par le test du $\chi^{2}$. 
Ensuite, différents tests non paramétriques : test de Kruskall et Wallis, test de Mann-Whitney, test de Friedman (Hollander ef Wolfe, 1973) ont été utilisés pour étudier statistiquement, chez les animaux porteurs de lésions cardiaques, les variations entre lots du nombre et de la sévérité de ces lésions.

\section{Résultats.}

Les lésions histiocytaires pouvant quelquefois masquer les lésions de nécrose, nous avons essayé, dans ce travail, de séparer ces dernières, non seulement des lésions purement histiocytaires mais aussi, pour une simplification de lecture, des lésions de nécrose associées à une histiocytose marquée (type $G_{2}$ ou $G_{3}$ ). Nous avons constaté que la plupart des lésions se situaient dans la région subépicardique.

\section{Résultats après 6 mois de régime (†abl. 1).}

Les différents tests statistiques appliqués aux données recueillies n'ont pas permis de mettre en évidence de différences significatives, à l'exception toutefois du nombre de rats porteurs de lésions cardiaques qui est plus élevé $\left(\chi^{2}, 0,05<P<0,1\right)$ avec un régime à 15 p. 100 d'huile (arachide ou colza Primor) qu'avec un régime à 5 ou à 10 p. 100, ainsi que du nombre total et de la sévérité des lésions, qui sont plus importants chez les animaux ingérant un régime à 15 p. 100 d'huile de colza Primor que chez ceux ingérant 15 p. 100 d'huile d'arachide (Mann-Whitney, 0,05<P<0,08).

\section{TABLEAU 1}

Résultats des éfudes morphologiques après 6 mois de mise au régime

\begin{tabular}{|c|c|c|c|c|c|c|}
\hline \multirow{2}{*}{$\begin{array}{c}\text { Nature de l'huile } \\
\text { Dose d'huile dans le régime (p. 100) ..... }\end{array}$} & \multicolumn{3}{|c|}{ Arachide } & \multicolumn{3}{|c|}{ Colza Primor } \\
\hline & 5 & 10 & 15 & 5 & 10 & 15 \\
\hline Nombre de rats/lot & 12 & 12 & 12 & 12 & 12 & 12 \\
\hline $\begin{array}{l}\text { Nombre de rats exempts de lésions cardia- } \\
\text { ques } \ldots \ldots \ldots \ldots \ldots \ldots \ldots \ldots \ldots \ldots \ldots\end{array}$ & 8 & 8 & 7 & 9 & 8 & 6 \\
\hline $\begin{array}{l}\text { Nombre de rats porteurs de lésions « dou- } \\
\text { teuses } " \ldots \ldots \ldots \ldots \ldots \ldots \ldots \ldots \ldots \ldots\end{array}$ & 1 & 2 & 0 & 0 & 1 & 0 \\
\hline $\begin{array}{l}\text { Nombre de rats porteurs de lésions cardia- } \\
\text { ques } \ldots \ldots \ldots \ldots \ldots \ldots \ldots \ldots \ldots \ldots \ldots\end{array}$ & 3 & 2 & 5 & 3 & 3 & 6 \\
\hline Nombre moyen de lésions ( ${ }^{(1)} /$ rat atteint & $13 / 3$ & $4 / 2$ & $16 / 5$ & $20 / 3$ & $17 / 3$ & $56 / 6$ \\
\hline $\begin{array}{l}\text { Nombre moyen de lésions nécrotiques }\left({ }^{2}\right) / \\
\text { rat atteint. } \ldots \ldots \ldots \ldots \ldots \ldots \ldots \ldots \ldots\end{array}$ & $1 / 1$ & $4 / 2$ & $10 / 3$ & $14 / 2$ & $14 / 3$ & $39 / 6$ \\
\hline
\end{tabular}

(1) Lésions nécrotiques avec ou sans réaction histiocytaire.

$\left(^{2}\right.$ Lésions de type $V_{1} G_{1}, V_{2} G_{1}$, ou $V_{3} G_{1}$ (lésions nécrotiques à faible réaction histiocytaire). 
2. Résultats après 1 an de régime (tabl. 2).

II n'y a pas de différence quant au nombre de rats porteurs de lésions. L'augmentation du nombre total de lésions ou des lésions nécrotiques constatée chez les animaux des lots colza Primor n'est pas significative.

TABLEAU 2

Résultałs de éfudes morphologiques oprès 1 on de mise au régime

\begin{tabular}{|c|c|c|}
\hline Nature de l'huile & Arachide & Colza Primor \\
\hline Dose d'huile dans le régime (p. 100) $\ldots$ & 5 & 5 \\
\hline Nombre de rats/lot $\ldots \ldots \ldots \ldots \ldots \ldots \ldots \ldots \ldots$ & 12 & 12 \\
\hline Nombre de rats exempts de lésions ...... & 4 & 3 \\
\hline Nombre de rats porteurs de lésions « douteuses $» . . .$. & 1 & 2 \\
\hline Nombre de rats porteurs de lésions cardiaques ...... & 7 & 7 \\
\hline Nombre moyen de lésions $\left({ }^{1}\right) /$ rat atteint & $55 / 7$ & $76 / 7$ \\
\hline Nombre moyen de lésions, nécrotiques $\left({ }^{2}\right) /$ rat atteint. .. & $40 / 6$ & $60 / 6$ \\
\hline
\end{tabular}

(1) Lésions nécrotiques avec sous sans réaction histiocytaire.

(2) Lésions de type $V_{1} G_{1}, V_{2} G_{1}$ ou $V_{3} G_{1}$ (lésions nécrotiques à faible réaction histiocytaire).

3. Influence de la durée du régime sur le nombre d'animaux afteints ef la fréquence des lésions cardiaques.

Celle-ci n'a pu être déterminée que chez les rats soumis aux régimes à 5 p. 100 d'huile. On constate que le nombre d'animaux porteurs de lésions est significativement plus élevé $\left(\chi^{2}, P<0,05\right)$ après un an d'expérience qu'après 6 mois ; il n'y a pas de différence en ce qui concerne la fréquence des lésions cardiaques.

\section{Discussion et conclusion.}

Cette expérience s'ajoute aux nombreuses études effectuées, ces dernières années, sur les effets cardiopathogènes comparés des huiles de colza pauvres en acide érucique et d'autres corps gras alimentaires (Rocquelin, 1977). Nos résultats seront donc interprétés dans ce contexte général.

Contrairement à nos observations antérieures, nous constatons ici que le nombre de rats, ingérant de l'huile d'arachide, qui présentent des lésions cardiaques n'est pas nul et qu'il est voisin de celui obtenu chez les rats des lots huile de colza Primor. Il est difficile d'expliquer l'origine de cette divergence, mais il est possible que la méthode de lecture des coupes, appliquée dans cette expérience, nous ait conduits à prendre en compte plus de lésions suspectes ou peu sévères et que la multiplication des coupes transversales, plus nombreuses que celles effectuées précédemment, ait entraîné une 
augmentation du nombre des lésions recensées. D'autres facteurs, incontrôlés, sont peut-être aussi à l'origine de ces divergences. Quoi qu'il en soit, les différences d'effets entre l'huile d'arachide ef l'huile de colza Primor, éłablies sur la fréquence du nombre de rats atteints de lésions, sont peu sensibles et non significatives. C'est au niveau du nombre et de la sévérité des lésions cardiaques que l'on retrouve, comme dans nos études antérieures, un effet cardiopathogène du régime contenant 15 p. 100 d'huile de colza Primor plus marqué que celui du régime contenant de l'huile d'arachide. Les tendances non significatives entre les 2 types d'huile aux taux de 5 et $10 \mathrm{p}$. 100 dans le nombre moyen de lésions nécessiteraient un plus grand nombre d'animaux éłudié.

Les résultats après 1 an de régime à 5 p. 100 confirment la présence de lésions cardiaques chez les rats arachide et colza Primor. Bien qu'il n'existe pas de différence statistiquement significative entre les 2 lots, on remarque une tendance à un plus grand nombre de lésions pour le lot colza Primor. Il est sans doute regrettable que des rats soumis pendant un an à des régimes à 10 ef 15 p. 100 d'huile n'aient pas pu être mis en expérience.

Nous avons, par ailleurs, décelé un effet de la dose de lipides dans le régime sur le nombre de rats porteurs de lésions cardiaques. Ce résultat intéressant confirme ceux de Hulan, Kramer et Corner (1977) et mériterait d'être complété par d'autres travaux.

La présente étude, ainsi que celles d'autres auteurs, (Beare-Rogers, Nera et Heggtveit, 1974 ; Kramer et al., 1975 ; Vles et al., 1976) montrent que la variabilité du nombre de rats « témoins » porteurs de lésions cardiaques peut être grande d'une expérience à une autre et qu'elle ne permet pas toujours de tirer des conclusions nettes. Dans le cas présent, il nous aurait fallu sans doute un nombre de rats plus important dans chaque lot pour y parvenir.

Cette expérience ne nous permet done pas de répondre clairement à la question qui nous était posée : existe-t-il une dose d'huile de colza Primor qui, dans le régime du Rat, n'engendre pas de lésions cardiaques?

Effets physiologiques comparés des huiles de colza et d'arachide.

ATP-INRA, 1974-1976.

\section{Références}

BEARE-ROGERS J. L.. NERA E. A., HEGGTVEIT H. A., 1974. Myocardial alteration in rats fed rapeseed oils containing high or low levels of erucic acid. Nutr. Metabol., 17, 213-222.

HOLLANDER M., WOLFE O. A., 1973. Non parametric stafistical methods. John Wiley and Sons, New York.

HULAN H. W., KRAMER J. K. G., CORNER A. H., 1977. Myocardial lesions in rats fed rapeseed oil. 1. Influence of strain of rats. Con. J. Physiol. Pharmacol., 55, 258-264.

KRAMER J. K. G., HULAN H. W., MAHADEVAN S., SAUER F. D., 1975. Brassica Campestris var. Span : II. Cardiopathogenicity of fractions isolated from span rapeseed oil when fed to male rats. Lipids, 10, 511-516.

ROCQUELIN G., 1977. Connaissances actuelles des effets des huiles de crucifères à faible teneur en acide érucique sur le myocarde. Méd. Nufr., 13, 269-275.

VLES R. O., BIJSTER G. M., KLEINEKOORT J. S. W., TIMMER W. G., ZAALBERG J., 1976. Nutritional status of low erucic acid rapeseed oils. Fette Seif. Anstrich., 78, 128-131. 